\title{
Caracterizando aspectos do sistema de informação contábil na gestão de custos: Um estudo empírico no âmbito do agronegócio
}

\author{
Antonio André Cunha Callado \\ Doutorado em Administração pela Universidade Federal do Paraíba - UFPB \\ Professor da Universidade Federal do Pernambuco - UFPE \\ E-mail: andrecallado@yahoo.com.br \\ Aldo Leonardo Cunha Callado \\ Doutorando em Agronegócios pela Universidade Federal do Rio Grande do Sul - \\ UFRGS \\ Professor da Universidade Federal do Paraíba - UFPB \\ E-mail: aldocallado@yahoo.com.br \\ Maria Conceição Melo Silva \\ Doutoranda em Administração pela Universidade Federal de Pernambuco - UFPE \\ Professora da Universidade Federal do Pernambuco - UFPE \\ E-mail:ceica@infonet.com.br \\ Luiz Carlos Miranda \\ Doutorado em Ph. D. Program in Agribusiness - University of Illinois \\ Professor da Universidade Federal do Pernambuco - UFPE \\ E-mail:Ic-miranda@uol.com.br
}

\section{RESUMO}

O objetivo deste trabalho consistiu em identificar a dinâmica das relações entre fatores que compõem o sistema de informação contábil sobre custos em agroindústrias pernambucanas. Foram pesquisadas 88 empresas e analisadas 10 variáveis. Foram utilizadas três abordagens metodológicas multivariadas complementares: a análise de agrupamentos, o escalonamento multidimensional e a análise fatorial. Foi identificado um agrupamento principal composto pelas variáveis referentes ao uso de formulários específicos, ao processo de escrituração e ao processo de registro dos custos. A análise fatorial demonstrou que as variáveis descartadas pelas técnicas anteriores como pertencentes ao núcleo não podem ser consideradas como exógenas ao fenômeno estudado.

Palavras-chave: Gestão de custos. Sistema de informação contábil. Estudo empírico. Agronegócio. Contabilidade gerencial.

Characterizing the main aspects of the acconting information system in costs management: An empiric study in agribusiness 
Caracterizando aspectos do sistema de informação contábil na gestão de custos: Um estudo empírico no âmbito do agronegócio

Antonio André Cunha Callado, Aldo Leonardo Cunha Callado, Maria Conceição Melo Silva, Luiz Carlos

Miranda

\section{ABSTRACT}

The objective of this article was identifying the dynamics relations between accounting information system factors related to costs in agribusiness from Pernambuco. The research considered 88 companies and analyzed 10 variables. Three multivariate approaches were used: cluster analysis, multimensional scaling, factor analysis. One main cluster identified was composed by the use of reports as well as the recording process of costs data. Factor analysis demonstrated that the other variables dismissed by the previous tools cannot be considered as exogenous to the relations framework.

Keywords: Costs management. Accounting information system. Empiric study. Agribusiness. Accounting. Managerial accounting.

\section{INTRODUÇÃO}

Os custos operacionais e de produção das organizações agroindustriais, embora sejam compostos basicamente pelos mesmos elementos do custo de outros tipos de empresas, são caracterizados pelas peculiaridades inerentes ao próprio setor de atividade econômica no qual elas estejam inseridas.

Franco (1988) afirma que as organizações agroindustriais possuem características próprias de formação do custo, conforme a natureza da atividade econômica que for explorada.

A contabilidade de custos é responsável pelo registro e pelo controle das operações, para determinação dos custos e estimação dos resultados de produção agrícola, zootécnica e agro-industrial. Valle e Aloe (1981) revelam a importância da aplicação prática dos estudos sobre o comportamento dos custos nas empresas rurais, destacando sua relevância como um eficiente termômetro.

Para Santos, Marion e Segatti (2002), um sistema gerador de informações sobre os custos agroindustriais é relevante como instrumento gerencial fundamental para auxiliar a tomada de decisão, bem como ressaltam que um sistema de custos é um conjunto de procedimentos administrativos que registra, de forma sistemática e contínua, a efetiva remuneração dos fatores de produção empregados nos serviços rurais. 
Caracterizando aspectos do sistema de informação contábil na gestão de custos: Um estudo empírico no âmbito do agronegócio

Antonio André Cunha Callado, Aldo Leonardo Cunha Callado, Maria Conceição Melo Silva, Luiz Carlos

Miranda

Como parte de um sistema de informações, a contabilidade de custos é desenvolvida através de coleta e processamento de dados que culmina com a produção e distribuição de informações na forma de relatórios contábeis. Esses relatórios devem garantir o fluxo contínuo de informações, permitindo a avaliação da situação atual e a comparação com o planejamento, para identificar e controlar os desvios, assim como suas causas.

Os produtores rurais podem utilizar esta informação como elemento auxiliar de sua administração para escolher as criações e as práticas agrícolas que deverão ser adotadas nos próximos períodos.

Segundo Oliveira Filho e Nerger (2004), dentro da agricultura brasileira impera a necessidade do agricultor produzir em condições cada vez mais competitivas, o que passa necessariamente pela racionalização dos custos, uma vez que, na ausência de interferências governamentais, a competição interna e externa passa a ser dada pelo uso mais eficiente dos recursos disponíveis, como a análise econômica e financeira de uma propriedade rural. Portanto, o conhecimento e domínio dessas variáveis passam a desempenhar um papel crucial para o sucesso da atividade agrícola.

Pode-se afirmar que as informações relativas aos custos de produção agroindustrial são fundamentais no que se refere à gestão e à competitividade no agronegócio contemporâneo. A contínua busca por maiores padrões de qualidade em comunhão a rígidos controles de custos, estão situados como pilares referenciais cada vez mais desejados pelos produtores que buscam expandir seus mercados consumidores, bem como obter maiores lucros.

Dentro da literatura acadêmica não há registro de pesquisas anteriores que tenham investigado relações dinâmicas entre os fatores que compõem um sistema de informação contábil sobre custos no âmbito do agronegócio através de uma abordagem multivariada.

A presente pesquisa encontra-se vinculada a um projeto financiado pelo CNPq, e o objetivo deste trabalho consiste em identificar a dinâmica das relações entre fatores 
Caracterizando aspectos do sistema de informação contábil na gestão de custos: Um estudo empírico no âmbito do agronegócio

Antonio André Cunha Callado, Aldo Leonardo Cunha Callado, Maria Conceição Melo Silva, Luiz Carlos

Miranda

que compõem o sistema de informação contábil sobre custos em organizações agroindustriais pernambucanas.

\section{GESTÃO DE CUSTOS EM ORGANIZAÇÕES AGROINDUSTRIAIS}

A contabilidade de custos no âmbito das empresas que atuam no agronegócio é uma ferramenta gerencial considerada como complexa em sua execução. Pode ser observado que a gestão de organizações agroindustriais se desenvolve dentro de critérios tradicionais e que apresentam um padrão de desempenho restrito considerando seu potencial global. A contabilidade rural é quase sempre utilizada para atender as finalidades fiscais, não possuindo grande interesse por uma aplicação gerencial.

Hofer et al., (2004) apresentam que os empresários rurais, normalmente, não costumam utilizar a contabilidade, especialmente a de custos como um instrumento de grande valia para esses produtores conduzirem seus empreendimentos de forma adequada, já que muitas empresas rurais não são estruturadas formalmente e nem registradas como Pessoa Jurídica, com contabilidade escriturada.

Sobre as principais dificuldades relacionadas à aplicação da contabilidade de custos como suporte gerencial, Crepaldi (1998) afirma que essa característica não é atributo apenas de pequenas propriedades rurais, prevalecendo também entre as médias e grandes, com economia de mercado e elevados níveis de renda.

A gestão de custos no agronegócio enfrenta uma limitação organizacional para sua plena implantação e desenvolvimento que é a dificuldade sobre o rigor no controle dos elementos de custo. Uma correta apropriação dos custos de cada produto, principalmente considerando os gastos gerais, deve permitir o rateio dos custos agroindustriais pelos diversos produtos e serviços prestados.

Sobre a importância de um sistema de custos, Marion (2000) destaca seus objetivos dentro da empresa afirmando que refletem sua importância como ferramenta básica para a administração de qualquer empreendimento, especialmente na 
Caracterizando aspectos do sistema de informação contábil na gestão de custos: Um estudo empírico no âmbito do agronegócio Antonio André Cunha Callado, Aldo Leonardo Cunha Callado, Maria Conceição Melo Silva, Luiz Carlos Miranda

agropecuária, onde os espaços de tempo entre produção e vendas, ou seja, entre custos e receitas, fogem à simplicidade de outros tipos de negócios.

Comentando sobre a participação da contabilidade de custos na gestão do agronegócio, Pereira (1996) afirma que a eficiência e a eficácia dos controles de custos para a gestão econômica envolvem necessariamente o estabelecimento dos padrões de eficiência e consequentemente os padrões de custos das atividades na agropecuária, de forma a possibilitar a formulação de orçamentos que atendam às tarefas de planejamento e controle.

Santos, Marion e Segatti (2002), destacam que um sistema de custos é um conjunto de procedimentos administrativos que registra, de forma sistemática e contínua, a efetiva remuneração dos fatores de produção empregados nos serviços rurais.

Os sistemas de custos são provedores de informações para que a gerência possa tomar as decisões mais corretas possíveis, permitindo identificação de gastos que estejam reduzindo a lucratividade da exploração. Esses relatórios devem garantir o fluxo contínuo de informações, permitindo a avaliação da situação atual e a comparação com o planejamento, para identificar e controlar os desvios, assim como suas causas.

Para Crepaldi (1998), um sistema contábil eficiente aliado ao bom senso do administrador deve proporcionar um diagnóstico realista, com a localização de pontos fracos e fortes de cada atividade produtiva e da empresa como um todo.

Santos, Marion e Segatti (2002), apresentam quatro objetivos de sistemas de custos, a saber:

a) auxiliar a administração na organização e controle da unidade de produção, revelando ao administrador as atividades de menor custo, as mais lucrativas, as operações de maior e menor custo e as vantagens de substituir por outras;

b) permitir uma correta valorização dos estoques para apuração dos resultados obtidos em cada cultivo ou criação; 
Caracterizando aspectos do sistema de informação contábil na gestão de custos: Um estudo empírico no âmbito do agronegócio Antonio André Cunha Callado, Aldo Leonardo Cunha Callado, Maria Conceição Melo Silva, Luiz Carlos Miranda

c) oferecer bases consistentes e confiáveis para projeção dos resultados e auxiliar o processo de planejamento rural, principalmente quando 0 administrador precisa decidir o que plantar, quando plantar e como plantar;

d) orientar os órgãos públicos na fixação de medidas, como garantia de preços mínimos, incentivos à produção de determinado produto em escala desejada, estabelecimento de limites de crédito.

No atual contexto econômico, os segmentos mais dinâmicos do agronegócio são constantemente desafiados a aprimorar seus conhecimentos sobre a administração, procurando, não só alcançar elevados níveis de produtividade, mas também saber como gerenciar a produtividade obtida.

Rauber et al., (2005) apresentam que o empresário rural deve buscar meios para diminuir o custo da produção, evitar desperdícios e melhorar o planejamento e controle de suas atividades, o que possibilita gerar informações precisas e oportunas sobre a situação real da produção e do resultado das culturas de sua propriedade.

\section{SISTEMA DE INFORMAÇÃO CONTÁBIL E DE CUSTOS}

Magalhães e Lunkes (2000) determinam que para uma gestão empresarial ser eficiente, é necessário um conjunto completo de relatórios (dados processados e agrupados), gerados mediante uma rede de processamentos integrantes de vários subsistemas, entre os quais o sistema contábil, que irão auxiliar nos vários níveis do processo decisório (planejamento estratégico, gerencial e operacional).

Stair e Reynolds (2006) afirmam que um sistema de informação contábil executa inúmeras atividades importantes, fornecendo informações detalhadas sobre contas a pagar, contas a receber, folha de pagamento, orçamentos, além de outras aplicações, com o sistema de custos responsável pelos gastos e receitas de uma empresa.

Um sistema contábil é alimentado por informações oriundas do ambiente interno, tais como: das áreas de operação, produção, estoque, pessoal; e do ambiente externo como: vendedores, clientes, empresas de auditoria, fornecedores, parceiros de 
Caracterizando aspectos do sistema de informação contábil na gestão de custos: Um estudo empírico no âmbito do agronegócio

Antonio André Cunha Callado, Aldo Leonardo Cunha Callado, Maria Conceição Melo Silva, Luiz Carlos

Miranda

negócios, entidades do governo, etc., permitindo que sejam feitas as transações contábeis referentes a livro-razão, contas a pagar e a receber, relatórios periódicos que permitirão um controle e gerenciamento sobre o orçamento empresarial, análise e controle de custos, auditoria e gerenciamento de impostos (Magalhães; Lunkes, 2000; Turban, Rainer jr.; Potter, 2003).

Riella et al., (2003) sustentam que com sistemas de informação as empresas possuem um diferencial que garante a redução de custos, maior agilidade nos processos e agregam valor às atividades empresariais. Os sistemas são melhorados no sentido de retornar informações mais completas ao usuário, mais rápidas e com maior precisão. Os processos são otimizados, garantindo maior ganho para a empresa.

Para Anthony (1972), um sistema contábil adequado deve ter os seguintes objetivos: processar a informação de forma eficiente; gerar relatórios prontamente; assegurar um alto grau de precisão; e minimizar a possibilidade de roubo, fraude ou representação errônea.

A última característica apresentada está associada ao grau de confiabilidade das informações geradas, ou seja, o controle interno da informação contábil. Assim, os resultados decorrentes da execução devem ser avaliados em relação aos planejados e os desvios apurados e corrigidos (BIO, 1989).

Guerreiro (2002) destaca que a informação útil é aquela que atende as necessidades específicas dos gestores, segundo as áreas que atuam, operações que desenvolvem e conceitos que Ihes façam sentido lógico. Os sistemas de informações contábeis devem ser configurados de forma a atender eficientemente as necessidades informativas de seus usuários, bem como incorporar conceitos, políticas e procedimentos que motivem e estimulem o gestor a tomar as melhores decisões para a empresa.

Alcançar melhores resultados, naturalmente, significa estabelecer um correto planejamento das atividades e correspondente controle, passando-se a obter informações da maior relevância para a apreciação do que a empresa realiza e do que pretende realizar. Neste contexto, o sistema de custos tem um importante papel, visto 
Caracterizando aspectos do sistema de informação contábil na gestão de custos: Um estudo empírico no âmbito do agronegócio

Antonio André Cunha Callado, Aldo Leonardo Cunha Callado, Maria Conceição Melo Silva, Luiz Carlos

Miranda

que para Pompermeyer (1999) esse sistema representa os meios que a empresa utilizará para coletar e sistematizar os dados de que necessita para produzir informações gerenciais úteis para toda a organização e seus níveis hierárquicos.

Os sistemas de custos se desenvolveram a partir da necessidade de aprimorar os métodos de avaliação dos estoques, diante de um volume de produtos em elaboração que exigia acurada medição e conseqüente valorização dos inventários, fazendo com que as empresas reconhecessem sua importância para o gerenciamento dos negócios e tomada de decisões, mediante as informações confiáveis para a projeção de resultados e para estudos econômicos. Observa-se, então, que a contabilidade de custos, a partir daí passa a oferecer elementos de medição e análise para alcançar objetivos administrativos e econômicos, além dos financeiros (Martins, 2003).

Desta forma, os fundamentos do sistema de custo objetivam alternadamente ou simultaneamente a avaliação de estoques, o controle gerencial e a tomada de decisão (Leone, 2001). Para tal, é importante que o sistema de gestão de custos prescinda de um patamar mínimo de organização dos subsistemas de apoio como: materiais, recursos humanos, finanças, produção e contabilidade. Portanto, tais subsistemas deverão ser articulados em torno dos dados que fornecerão ao sistema de custos, de forma sistematizada e organizada (Pompermeyer, 1999).

A tarefa de implantação de um sistema de gestão requer cuidados básicos, uma vez que para isso é exigida uma abordagem técnica específica, uma sensibilização das relações humanas e uma adequação da tecnologia da informação (Stair e Reynolds, 2006). É responsabilidade da empresa considerar que aspectos são relevantes na sua adoção para que se possa munir de informações que vão gerar conhecimento do negócio e melhores lucros.

Segundo Souza et al., (2004), com o uso de Sistemas de Informações, busca-se a redução de custos tanto para clientes internos como externos, e as medidas típicas para custos são o preço de aquisição, custo de propriedade e tempo gasto no processo. 
Caracterizando aspectos do sistema de informação contábil na gestão de custos: Um estudo empírico no âmbito do agronegócio Antonio André Cunha Callado, Aldo Leonardo Cunha Callado, Maria Conceição Melo Silva, Luiz Carlos Miranda

Os mesmos autores observam que a informação é capaz de alterar a relação de custos em qualquer parte da cadeia de valor de uma empresa.

\section{METODOLOGIA UTILIZADA}

\subsection{Natureza do Estudo}

O presente estudo caracteriza-se como uma pesquisa exploratória. Segundo Gil (1996), a pesquisa exploratória tem como objetivo proporcionar maior familiaridade com o problema, com vistas a torná-lo mais explícito ou a construir hipóteses para estudos futuros.

Segundo Vergara (2003), a investigação exploratória é realizada em área na qual há pouco conhecimento acumulado e sistematizado que, por sua natureza de sondagem não comporta hipóteses que, todavia, poderão surgir durante ou ao final da pesquisa.

Quanto à finalidade das pesquisas exploratórias, Gil (1999) apresenta que elas buscam desenvolver, esclarecer e modificar idéias, tendo em vista a formulação de problemas mais precisos ou hipóteses pesquisáveis para estudos posteriores, sendo que as pesquisas dessa natureza habitualmente envolvem levantamento bibliográfico e documental para sua realização.

\subsection{Definição da amostra da pesquisa}

Para Leite (1979), na definição de universo pode-se delimitar o campo de pesquisa em termos temporais, geográficos, setoriais ou qualquer outra dimensão, cabíveis com base na disponibilidade de dados ou com o fundamento nos objetivos e nos custos de execução da pesquisa.

O Estado de Pernambuco possui vários eixos de produção agroindustrial. De acordo com o Guia de Pernambuco (2005), três sub-regiões se destacam pela produção agroindustrial. A primeira se localiza na Zona da Mata e caracteriza-se pela cultura da cana-de-açúcar, pela avicultura, pela pecuária de corte, pela equinocultura e 
Caracterizando aspectos do sistema de informação contábil na gestão de custos: Um estudo empírico no âmbito do agronegócio Antonio André Cunha Callado, Aldo Leonardo Cunha Callado, Maria Conceição Melo Silva, Luiz Carlos Miranda

pela floricultura. A segunda está situada no agreste meridional, e a produção de leite é uma das principais atividades agroindustriais desta região. A terceira se encontra às margens do Rio São Francisco. Esta sub-região se destaca na agricultura irrigada e pela fruticultura. As empresas agroindustriais sediadas nestas três sub-regiões configuram o universo da pesquisa.

Dada a impossibilidade de investigar todas as empresas que compõem o universo da pesquisa, definiu-se uma amostra representativa da população que atendesse tanto às restrições orçamentárias da pesquisa, como também aos requisitos científicos para que eles fossem válidos, ou seja, representação da população.

De acordo com Gil (1996), para que os dados obtidos em levantamento sejam significativos, é necessário que a amostra seja constituída por um número adequado de elementos e que representem as proporções existentes no universo pesquisado. Sendo assim, a amostra foi composta da seguinte maneira, conforme dados apresentados na tabela 1.

Tabela 1: Composição da amostra utilizada na pesquisa

\begin{tabular}{|c|c|}
\hline Sub-regiões & Amostra \\
\hline Zona da Mata & 48 \\
\hline Agreste Meridional & 24 \\
\hline Região do São Francisco & 16 \\
\hline Total & $\mathbf{8 8}$ \\
\hline
\end{tabular}

Fonte: Pesquisa de campo, 2006.

O critério utilizado para identificar as empresas inseridas na amostra foi a acessibilidade, uma vez que a distribuição geográfica poderia inviabilizar a coleta dos dados. Portanto, a amostra da pesquisa foi composta por 88 agroindústrias.

\subsection{Variáveis Investigadas}

Diante do problema levantado e do objetivo proposto para esta pesquisa, foram estudadas as seguintes variáveis:

- Tamanho das empresas

- Sistemas de custos 
Caracterizando aspectos do sistema de informação contábil na gestão de custos: Um estudo empírico no âmbito do agronegócio Antonio André Cunha Callado, Aldo Leonardo Cunha Callado, Maria Conceição Melo Silva, Luiz Carlos

- Valoração dos estoques

- Uso de formulários

- Processamento do registro de custos

- Plano de contas dos dados de custos

- Frequência da emissão dos relatórios

- Cálculo do lucro

- Atualização dos dados de custos

- Escrituração dos dados de custos

\subsection{Método de coleta e análise dos dados}

O instrumento utilizado para a coleta de dados foi o questionário. Sobre o questionário, Chizzotti (1991) indica que ele consiste em um conjunto de questões préelaboradas, sistemática e seqüencialmente dispostas em itens que constituem o tema da pesquisa, com o objetivo de suscitar dos informantes respostas por escrito ou verbalmente sobre o assunto que os informantes saibam opinar ou informar.

Esta afirmativa é corroborada por Gil (1996), ao indicar que a sua elaboração consiste basicamente em traduzir os objetivos específicos da pesquisa em itens bem redigidos. Para Marconi e Lakatos (1996), a utilização do questionário como instrumento de coleta de dados pode ser justificada em função das respostas rápidas, precisas e maior uniformidade na avaliação.

Os questionários foram aplicados por técnicos agropecuários selecionados e instruídos para coletar as informações de maneira objetiva, homogênea e sistemática, de modo que não houvesse interferências no processo de coleta dos dados ao longo das entrevistas realizadas.

Considerando que o objetivo proposto para esta pesquisa consiste em investigar as relações dinâmicas entre fatores que compõem o sistema de informação contábil sobre custos em organizações agroindustriais pernambucanas, a escolha do método de análise estatística utilizado precisou considerar esta especificidade. A análise multivariada é uma metodologia apropriada para atingir tal objetivo proposto. 
Caracterizando aspectos do sistema de informação contábil na gestão de custos: Um estudo empírico no âmbito do agronegócio Antonio André Cunha Callado, Aldo Leonardo Cunha Callado, Maria Conceição Melo Silva, Luiz Carlos Miranda

De acordo com Hair et al., (2005), a análise multivariada é a análise de múltiplas variáveis em um único relacionamento ou conjunto de relações.

Para a identificação da estrutura natural de relações entre as diversas variáveis, esta pesquisa considerou os seguintes procedimentos:

- Análise de agrupamentos

- Escalonamento multidimensional

- Análise fatorial

Os cálculos foram efetuados a partir do aplicativo computacional STATISTICA for windows. A operacionalização desta pesquisa considerou as variáveis estudadas expressas de maneira ordinal, representando matematicamente o crescente grau de intensidade do uso das informações sobre custos dentro de um fluxo de informações inseridas no processo gerencial das empresas investigadas.

\section{APRESENTAÇÃo E ANÁLISE dOS RESULTADOS}

Para a execução da análise de agrupamento foi primeiramente utilizado o procedimento de árvore de agrupamento (clustering tree). A regra de amalgamação adotada foi a simgle linkage e as distâncias foram calculadas a partir do procedimento city-block (manhattan). O resultado dessa análise é apresentado no gráfico 1. 
Caracterizando aspectos do sistema de informação contábil na gestão de custos: Um estudo empírico no âmbito do agronegócio Antonio André Cunha Callado, Aldo Leonardo Cunha Callado, Maria Conceição Melo Silva, Luiz Carlos

Miranda

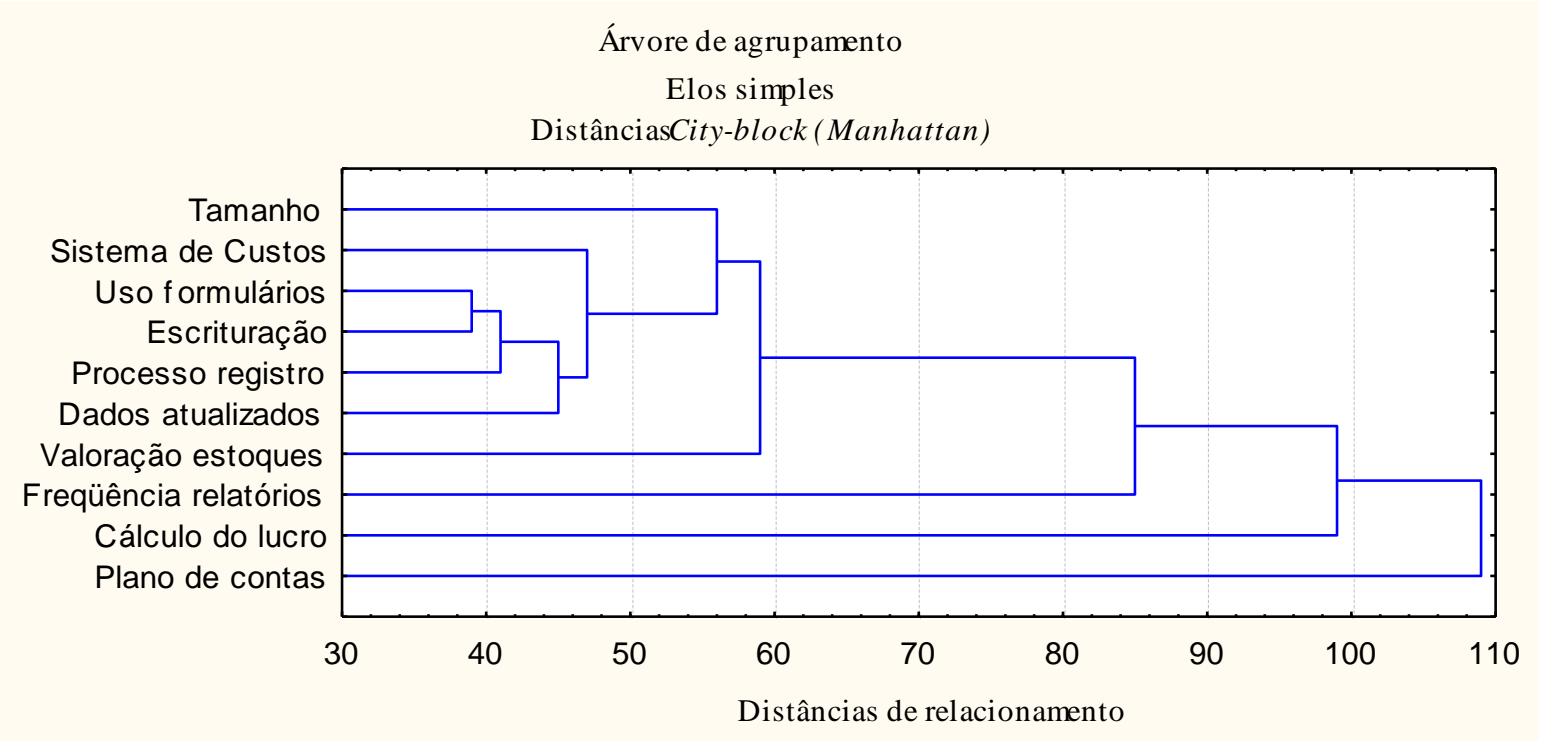

Gráfico 1 - Relação entre as variáveis através da análise de agrupamentos

Fonte: Cálculos próprios.

Número de variáveis: 10

Número de casos: 88

Regra de Amalgamação: Elos simples

Mensuração das Distâncias: Distâncias City-block (Manhattan)

O gráfico 1 representa os agrupamentos obtidos a partir das variáveis inseridas nesta pesquisa. A árvore de agrupamentos destaca a presença de um único grupo significativo de variáveis. Este agrupamento é composto pelas variáveis referentes ao uso de formulários específicos, ao processo de escrituração e ao processo de registro dos custos agroindustriais identificados.

As variáveis que mensuram o sistema de custos utilizado e o nível de atualização dos dados sobre custos agroindustriais podem ser relacionadas a este agrupamento, embora de maneira indireta.

A variável que representa o tamanho das empresas investigadas aparece quase sem possuir relações com este agrupamento. Este resultado pode indicar que o mais relevante sobre a eficácia de um sistema de informações sobre custos dentro do processo gerencial esteja relacionado à sua organização funcional propriamente dita, e não à estrutura financeira e operacional das empresas investigadas. 
Caracterizando aspectos do sistema de informação contábil na gestão de custos: Um estudo empírico no âmbito do agronegócio

Antonio André Cunha Callado, Aldo Leonardo Cunha Callado, Maria Conceição Melo Silva, Luiz Carlos

Miranda

Este grupo é caracterizado pela presença de variáveis que representam a intensidade de especialização e formalização dos parâmetros contábeis adotados pelos sistemas de custos utilizados pelas empresas investigadas. Nestes termos, na medida em que um sistema de informações que registra, classifica, analisa e disponibiliza relatórios sobre custos de maneira previamente categorizada, tende a suprir a tomada de decisões de maneira mais consistente e confiável.

Notadamente, pode-se atribuir que haja uma relação direta entre a especialização do profissional responsável pela análise de custos com a utilização de formulários especificamente desenvolvidos para atender a esta tarefa, os procedimentos de escrituração contábil dos dados sobre custos, sua contínua atualização e uso sistêmico.

Pode-se ainda destacar que outras variáveis aparentemente relacionadas ao processo de gestão de custos não tenham sido relacionadas ao núcleo central do agrupamento identificado. As variáveis que representam o método de valoração dos estoques, a frequência da elaboração e distribuição dos relatórios financeiros sobre custos, bem como os procedimentos de cálculo dos lucros se mostraram relativamente distanciadas do núcleo do agrupamento encontrado.

Outro aspecto relevante a ser comentado consiste no isolamento da variável que trata do plano de contas das empresas investigadas. Isto pode implicar em um descolamento da contabilidade geral das práticas cotidianas implementadas pela contabilidade de custos que é voltada para a gestão e para a tomada de decisão.

Em seguida, foi elaborado um gráfico bi-dimensional para ilustrar a escala multidimensional. A representação espacial da disposição das variáveis é importante para identificar sua disposição espacial e verificar se há similaridade com a composição do agrupamento obtido. Os resultados estão dispostos no gráfico 2 . 
Caracterizando aspectos do sistema de informação contábil na gestão de custos: Um estudo empírico no âmbito do agronegócio Antonio André Cunha Callado, Aldo Leonardo Cunha Callado, Maria Conceição Melo Silva, Luiz Carlos Miranda

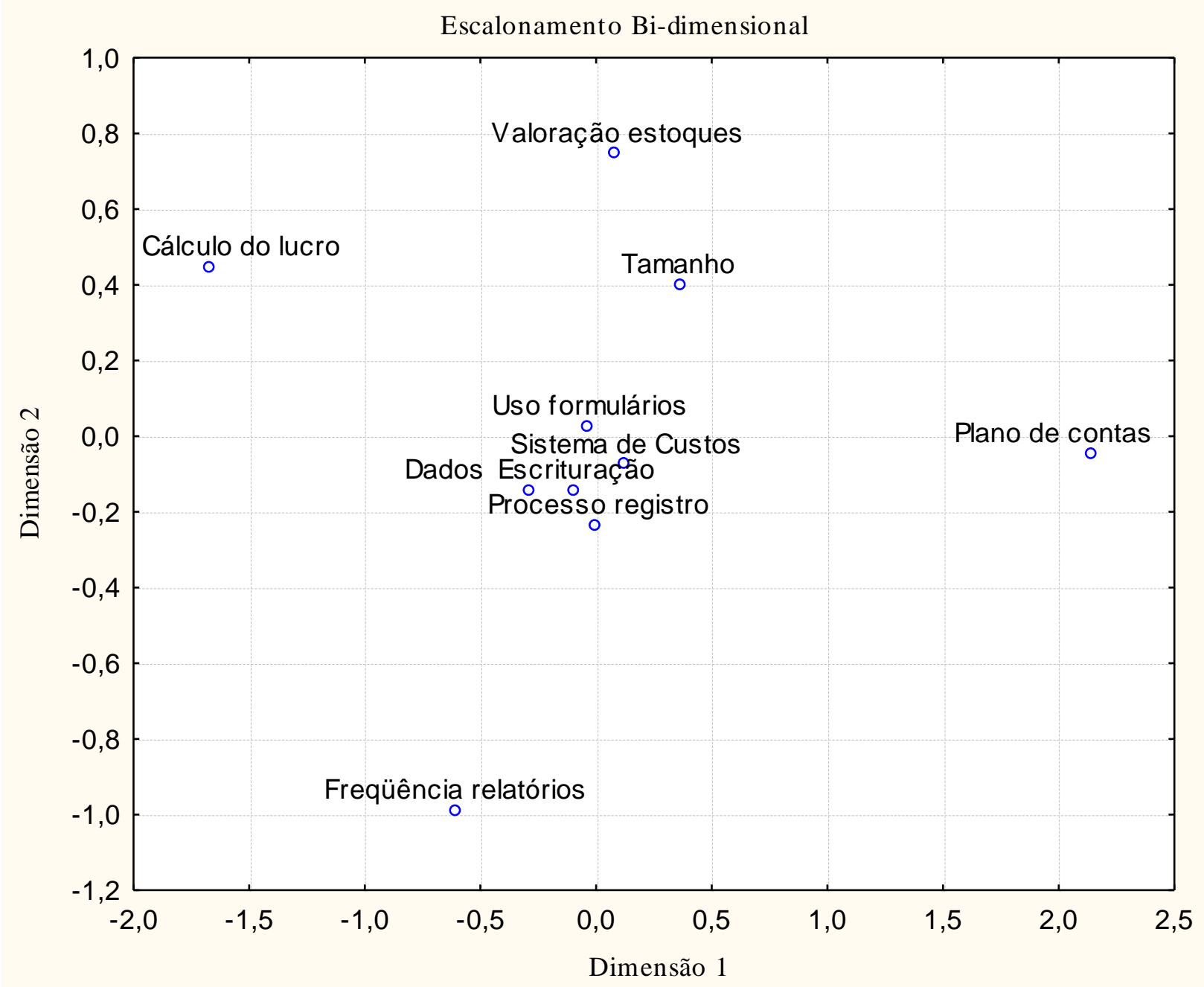

Gráfico 2 - Agrupamento das variáveis através do escalonamento bi-dimensional.

Fonte: Cálculos próprios.

Número de variáveis: 10

Número de dimensões: 2

Última interação computada: 104; Melhor iteração: 57

Alienação: 0,0645597

Estresse: 0,0367102

Os resultados apresentados através do gráfico bi-dimensional obtido corroboram a disposição das variáveis significativas dentro do agrupamento encontrado anteriormente, bem como confirma o relativo distanciamento das demais.

Cada uma das variáveis inseridas nesta investigação pode ser visualizada individualmente através da distribuição espacial gerada pelo escalonamento bi- 
Caracterizando aspectos do sistema de informação contábil na gestão de custos: Um estudo empírico no âmbito do agronegócio Antonio André Cunha Callado, Aldo Leonardo Cunha Callado, Maria Conceição Melo Silva, Luiz Carlos Miranda

dimensional. Estes resultados são estatisticamente significativos. Os níveis de alienação e estresse ficaram próximos a 0,05.

Pode-se observar que, de maneira semelhante ao resultado obtido pela árvore de agrupamento, as variáveis que representam o método de valoração dos estoques, a frequência da elaboração e distribuição dos relatórios financeiros sobre custos e os procedimentos de cálculo dos lucros se mostraram relativamente distanciadas do núcleo do agrupamento encontrado. Mais uma vez a variável que representa o tamanho das empresas investigadas aparece quase sem possuir relações com este agrupamento.

Com a finalidade de corroborar os resultados obtidos e dar maior precisão à representação gráfica do escalonamento multidimensional, os cálculos foram executados novamente para gerar um gráfico tri-dimensional. 
Caracterizando aspectos do sistema de informação contábil na gestão de custos: Um estudo empírico no âmbito do agronegócio Antonio André Cunha Callado, Aldo Leonardo Cunha Callado, Maria Conceição Melo Silva, Luiz Carlos Miranda

Escalonamento tri-dimensional

Configuração Final

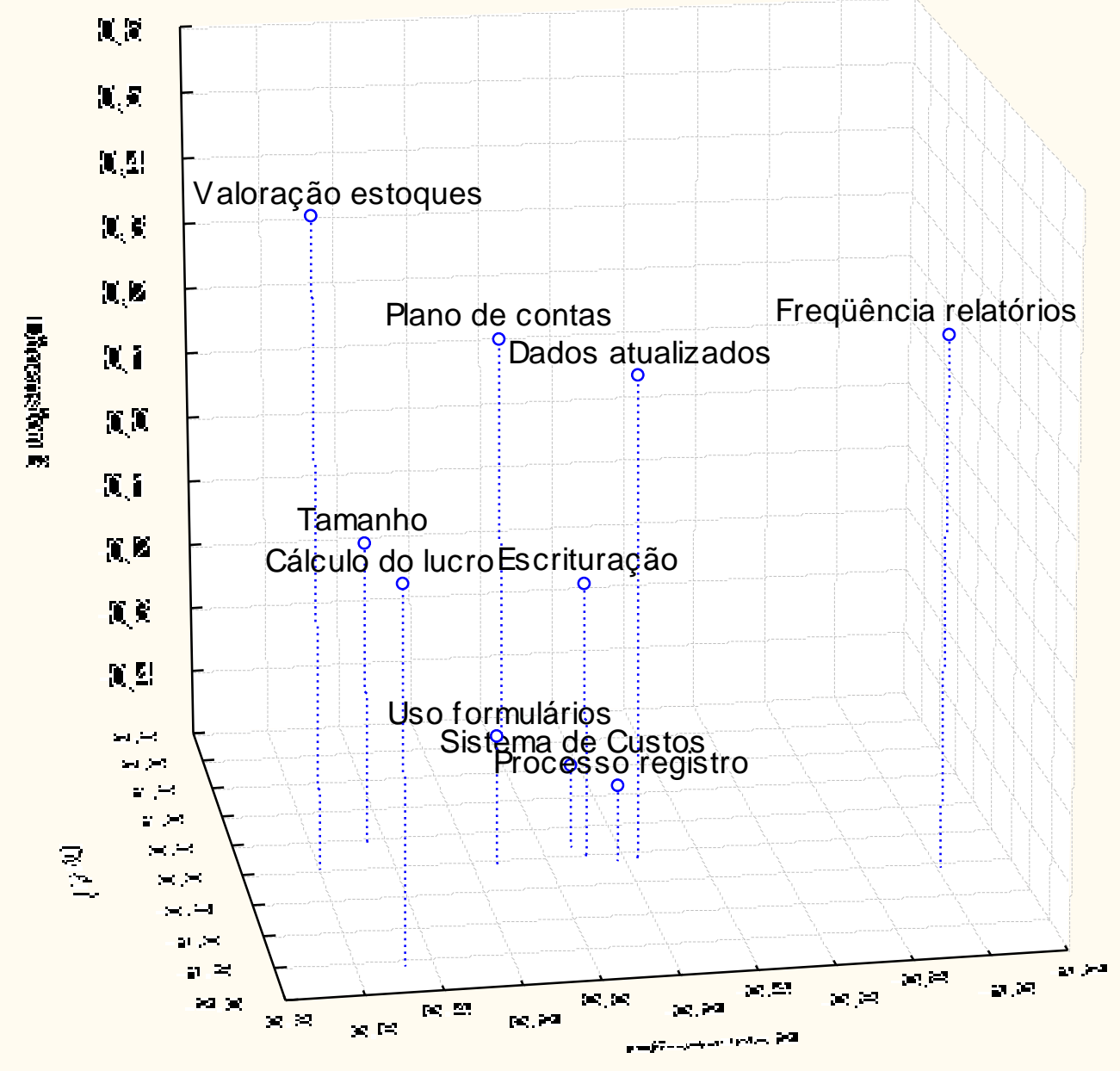

Gráfico 3 - Agrupamento das variáveis através do escalonamento tri-dimensional.

Fonte: Cálculos próprios.

Número de variáveis: 10

Número de dimensões: 3

Última interação computada: 97; Melhor iteração: 54.

Alienação: 0,0246922

Estresse: 0,0131614

O escalonamento tri-dimensional apresenta com maior nitidez a proximidade entre as variáveis que representam o uso de formulários específicos, o processo de registro dos dados sobre custos e os sistemas de custos adotados pelas empresas 
Caracterizando aspectos do sistema de informação contábil na gestão de custos: Um estudo empírico no âmbito do agronegócio Antonio André Cunha Callado, Aldo Leonardo Cunha Callado, Maria Conceição Melo Silva, Luiz Carlos

Miranda

investigadas. A variável que trata da escrituração se localiza um pouco acima dessas outras três variáveis. Ainda merece ser destacada a relativa distância apresentada pela variável que representa a atualização dos dados. Os níveis de alienação e estresse ficaram próximos a 0,01 .

O núcleo principal que representa as relações espaciais entre as variáveis investigadas é composto por formulários específicos, processos de registro dos custos e sistemas de custos. Este núcleo sintetiza a configuração básica de um sistema de informações voltado para atender as necessidades gerenciais e auxiliar o processo de tomada de decisão.

O distanciamento apresentado pelas demais variáveis se mostrou dentro da expectativa gerada a partir do escalonamento bi-dimensional.

Estes resultados confirmam que o uso de formulários específicos, processos de registro dos custos bem definidos e sistemas de custos voltados para a gestão estão, respectivamente, relacionadas entre si.

Finalmente, foi executada a análise fatorial. A configuração do cálculo ainda considerou a busca por três fatores, bem como um mínimo eigenvalue equivalente a 1 . Os resultados estão apresentados na tabela 2. 
Caracterizando aspectos do sistema de informação contábil na gestão de custos: Um estudo empírico no âmbito do agronegócio Antonio André Cunha Callado, Aldo Leonardo Cunha Callado, Maria Conceição Melo Silva, Luiz Carlos

Tabela 2 - Resultados da análise fatorial

\begin{tabular}{|c|c|c|}
\hline Variáveis & Fator 1 & Fator 2 \\
\hline \hline Tamanho & $-0,827968$ & $-0,405194$ \\
\hline \hline Sistema de custos & $-0,900483$ & $-0,123164$ \\
\hline \hline Valoração de estoques & $-0,789136$ & $-0,133907$ \\
\hline \hline Uso de formulários & $-0,926667$ & 0,019092 \\
\hline \hline Processo de registro & $-0,916797$ & 0,132254 \\
\hline Plano de contas & $-0,388152$ & $-0,789128$ \\
\hline \hline Freqüência dos relatórios & $-0,719317$ & 0,478531 \\
\hline Cálculo do lucro & $-0,634031$ & 0,430752 \\
\hline Atualização dos dados & $-0,898377$ & 0,099972 \\
\hline Escrituração & $-0,930818$ & 0,013201 \\
\hline Capacidade explicativa & $\mathbf{0 , 6 5 6 1 9 4}$ & $\mathbf{0 , 1 2 6 2 5 7}$ \\
\hline
\end{tabular}

Fonte: Cálculos próprios.

Número de variáveis: 10

Método: Componentes principais

Log (10) Determinante da matriz de correlação: $-4,9274$

Número de fatores extraídos: 2

Eigenvalues: Fator $1-6,56194$

Fator 2 - 1,26257

Pode-se observar que foram identificados dois fatores distintos. O primeiro fator é composto pelas variáveis que representam o tamanho, o sistema de custos, a valoração de estoques, o uso de formulários, o processo de registro, a freqüência dos relatórios, a atualização dos dados e a escrituração dos dados sobre custos. O segundo fator é composto pelo responsável pela variável que representa o plano de contas das empresas investigadas.

Os resultados obtidos através da análise fatorial demonstram que, mesmo considerando que o agrupamento de variáveis encontrado não seja composto por este elenco mais amplo, fica evidente que estes fatores referentes aos custos nas empresas agroindustriais são complementares entre si.

Estes resultados fornecem evidências empíricas significativas para a compreensão da relação dinâmica entre as variáveis investigadas e demonstra que a concepção de um sistema de informação gerencial é crucial para sua eficácia posterior. 
Caracterizando aspectos do sistema de informação contábil na gestão de custos: Um estudo empírico no âmbito do agronegócio Antonio André Cunha Callado, Aldo Leonardo Cunha Callado, Maria Conceição Melo Silva, Luiz Carlos Miranda

\section{CONCLUSÕES}

O objetivo deste trabalho consistiu em identificar a dinâmica das relações entre fatores que compõem o sistema de informação contábil sobre custos em organizações agroindustriais pernambucanas.

Para atingir o objetivo proposto, foram utilizadas os modelos e conceitos de análise multivariada composta pela análise de agrupamento, pelo escalonamento multidimensional e pela análise fatorial.

A árvore de agrupamentos destacou a presença de um único grupo significativo de variáveis. Este agrupamento é composto pelas variáveis referentes ao uso de formulários específicos, ao processo de escrituração e ao processo de registro dos custos agroindustriais identificados.

As variáveis que mensuram o sistema de custos utilizado e o nível de atualização dos dados sobre custos agroindustriais podem ser relacionadas a este agrupamento, embora de maneira indireta. A variável que representa o tamanho das empresas investigadas aparece quase sem possuir relações com este agrupamento.

O escalonamento multidimensional apresentou resultados semelhantes aos encontrados através da análise de agrupamentos, destacando a composição do núcleo central do agrupamento encontrado. Novamente, as variáveis que representam o método de valoração dos estoques, a frequência da elaboração e distribuição dos relatórios financeiros sobre custos e os procedimentos de cálculo dos lucros, se mostraram relativamente distanciadas do núcleo do agrupamento encontrado.

O escalonamento tri-dimensional apresentou com maior nitidez a proximidade entre as variáveis que representam o uso de formulários específicos, o processo de registro dos dados sobre custos e os sistemas de custos adotados pelas empresas investigadas. Os resultados obtidos apresentaram níveis de significância estatística próximos a 0,01 para os índices de alienação e de estresse referentes ao escalonamento multidimensional. Através deste resultado pode-se afirmar que a 
Caracterizando aspectos do sistema de informação contábil na gestão de custos: Um estudo empírico no âmbito do agronegócio Antonio André Cunha Callado, Aldo Leonardo Cunha Callado, Maria Conceição Melo Silva, Luiz Carlos Miranda

estrutura de relações espaciais entre as variáveis investigadas é significativa ao nível de $99 \%$.

Foram identificados dois fatores distintos na análise fatorial. O primeiro fator é composto pelas variáveis que representam o tamanho, o sistema de custos, a valoração de estoques, o uso de formulários, o processo de registro, a frequência dos relatórios, a atualização dos dados e a escrituração dos dados sobre custos. O segundo fator é composto pelo responsável pela variável que representa o plano de contas das empresas investigadas.

Estes resultados revelam indícios particulares referentes à caracterização do sistema de informação utilizado pelas organizações agroindustriais localizadas no Estado de Pernambuco que foram investigadas. Como estes resultados não podem ser generalizados para toda a população de empresas agroindustriais, estudos mais aprofundados poderão gerar informações mais detalhadas sobre a natureza das relações dinâmicas encontradas nesta pesquisa.

\section{REFERÊNCIAS}

ANTHONY, R. N. 1972. Contabilidade Gerencial. São Paulo, Atlas, 483 p.

BIO, S. R. 1989. Sistemas de informação: um enfoque gerencial. São Paulo, Atlas, 183 p.

CHIZZOTTI, A. 1991. A Pesquisa em Ciências Humanas e Sociais. São Paulo, Cortez, $164 \mathrm{p}$.

CREPALDI, S. A. 1998. Contabilidade Rural. 2ª ed. São Paulo, Atlas, 340 p.

FRANCO, H.1988. Contabilidade Industrial. 8ª ed. São Paulo, Atlas, 286 p.

GIL, A. C. 1999. Métodos e Técnicas de Pesquisa Social. 5ª ed. São Paulo, Atlas, 206 p.

GIL, A. C. 1996. Como Elaborar Projetos de Pesquisa. 3ª ed. São Paulo, Atlas, 159 p. 
Caracterizando aspectos do sistema de informação contábil na gestão de custos: Um estudo empírico no âmbito do agronegócio Antonio André Cunha Callado, Aldo Leonardo Cunha Callado, Maria Conceição Melo Silva, Luiz Carlos

Miranda

GUERREIRO, R. 2002. Um Modelo de Sistema de Informação Contábil para Mensuração do Desempenho Econômico das Atividades Empresariais. Caderno de Estudos FIPECAFI. 4:1-17.

GUIA DE PERNAMBUCO. Disponível em http://www.recifeguide.com/brasil/pernambuco/garanhuns.html, acesso em 28/02/2006.

HAIR, J. F. et al. 2005. Análise multivariada de dados. 5ª ed. Porto Alegre, Bookman, $593 \mathrm{p}$.

HOFER, Elza; et al. 2004. Custo de Produção para a Atividade da Pecuária Leiteira: Um Estudo de Caso. In: XI CONGRESSO BRASILEIRO DE CUSTOS. Porto Seguro, 2004. Anais... Porto Seguro, CD-ROM.

LEITE, J. A. A. 1979. Metodologia de Elaboração de Teses. São Paulo, Editora McGraw-Hill do Brasil, $122 \mathrm{p}$.

LEONE, G. S. G. 2001. Custos: um enfoque administrativo. 14a ${ }^{\mathrm{a}}$. ed. Rio de Janeiro: Fundação Getúlio Vargas, 576 p.

MAGALHÃES, A. de D. F. e LUNKES, I. C. 2000. Sistemas contábeis: o valor informacional da contabilidade nas organizações. São Paulo, Atlas, 192 p.

MARCONI, M. A. e LAKATOS, E. M. 1996. Técnicas de pesquisa: planejamento e execução de pesquisas, amostragens e técnicas de pesquisas, elaboração, análise e interpretação de dados. 3를. ed. São Paulo, Atlas, 205 p.

MARION, J. C. 2000. Contabilidade Rural. 6ª ed. São Paulo, Atlas, 278 p.

MARTINS, E. 2003. Contabilidade de Custos. 9ae ed. São Paulo, Atlas, 370 p.

OLIVEIRA FILHO, João Bento de; NERGER, Rodrigo. 2004. Gestão de Custos em Empresas de Agronegócios das Culturas de Soja e Milho no Cerrado Brasileiro. In: XI CONGRESSO BRASILEIRO DE CUSTOS, 2004, Porto Seguro. Anais... Porto Seguro: CD-ROM.

PEREIRA, E. 1996. Controladoria, gestão empresarial e indicador de eficiência em agribusiness._In: MARION, J. C. (coordenador). Contabilidade e controladoria em agribusiness. São Paulo, Atlas, 224 p.

POMPERMEYER, Cleonice Bastos. 1999. Sistemas de gestão de custos: dificuldades na implantação. Revista FAE, Curitiba, 2(3):21-28. 
RAUBER, Adriano José; et al. 2005. Gestão de Custos Aplicados à Atividade Rural para Culturas Temporárias: Um Estudo de Caso. In: XII CONGRESSO BRASILEIRO DE CUSTOS, 2005, Itapema. Anais... Itapema: CD-ROM.

RIELLA, Cláudio Gomes; et al. 2003. A Evolução dos Sistemas e a Nova Era Sistêmica: Rumo aos Sistemas Inteligentes. In: X CONGRESSO BRASILEIRO DE CUSTOS, 2003, Vitória. Anais. Vitória: CD-ROM.

SANTOS, G. J; MARION, J. C. e SEGATTI, S. 2002. Administração de Custos na Agropecuária. 3aㅡ ed. São Paulo, Atlas, 168 p.

SOUZA, Sérgio Alexandre; et al. 2004. Os Sistemas Integrados de Gestão - ERP: Aplicabilidade $\mathrm{Na}$ Gestão Contábil e na Controladoria das Empresas. In: XI CONGRESSO BRASILEIRO DE CUSTOS, 2004, Porto Seguro. Anais... Porto Seguro: CD-ROM.

STAIR, R. e REYNOLDS, G. W. 2006. Princípios de Sistemas de Informação. Rio de Janeiro, Thompson, $451 \mathrm{p}$.

TURBAN, E.; RAINER Jr. R.K. e POTTER, R. E. 2003. Administração de tecnologia da informação: teoria e prática. Rio de Janeiro, Elsevier, 624 p.

VALLE, F. e ALOE, A. 1981. Contabilidade Agrícola. 7ª ed. São Paulo, Atlas, 498 p.

VERGARA, S. C. 2003. Projetos e relatórios de pesquisa em administração. 4ª ed. São Paulo, Atlas, $96 \mathrm{p}$. 